\title{
Long-term azithromycin therapy in patients with severe COPD and repeated exacerbations
}

This article was published in the following Dove Press journal:

International Journal of COPD

3 September 201I

Number of times this article has been viewed

\section{Xavier Pomares' \\ Concepción Montón' \\ Mateu Espasa ${ }^{2}$ \\ Jordi Casabon' \\ Eduard Monsó ${ }^{1,3}$ \\ Miguel Gallego 1,3 \\ 'Pneumology Service, ${ }^{2}$ Laboratory Service, Corporació Parc Taulí, Universitat Autònoma de Barcelona, Sabadell, Spain; ${ }^{3}$ Ciber de Enfermedades Respiratorias - CibeRes, Bunyola, Spain}

Correspondence: Xavier Pomares Parc Taulí 2, 08208, Sabadell, Spain Tel +34937231010

Fax +34937162646

Email jpomares@tauli.cat
Background: The aim of this study was to determine whether long-term intermittent azithromycin therapy reduces the frequency of exacerbation in severe chronic obstructive pulmonary disease (COPD).

Methods: We retrospectively investigated the clinical benefits of long-term azithromycin (500 mg orally three times per week) over 12 months in patients with severe COPD and a minimum of four acute exacerbations (AECOPD) per year or chronic bronchial colonization by Pseudomonas aeruginosa, comparing the number of AECOPD, hospitalizations due to respiratory disease, days of hospital stay, and bacterial infections during azithromycin treatment and in the year prior to this therapy.

Results: Twenty patients who completed the 12-month treatment period were analyzed. No clinically significant adverse events were observed during azithromycin treatment. Compared with baseline data, azithromycin therapy significantly reduced the number of AECOPD $(2.8 \pm 2.5$ versus $6.8 \pm 2.8, P<0.001)$, hospitalizations $(1.4 \pm 1.5$ versus $3.6 \pm 1.4, P<0.001)$, and cumulative annual days of hospital stay $(25 \pm 32.2$ versus $43.7 \pm 21.4, P=0.01)$. The improvement was particularly significant in patients with exacerbations caused by common potentially pathogenic microorganisms, who had 70\% fewer AECOPD and hospitalizations. Patients colonized by P. aeruginosa had reductions of $43 \%$ in AECOPD and $47 \%$ in hospitalizations.

Conclusion: Long-term azithromycin is well tolerated and associated with significant reductions in AECOPD, hospitalizations, and length of hospital stay in patients with severe COPD.

Keywords: azithromycin, chronic obstructive pulmonary disease, exacerbation, macrolides

\section{Introduction}

Acute exacerbations of chronic obstructive pulmonary disease (COPD) are a frequent event during evolution of the disease, ${ }^{1}$ and the mortality risk increases with its frequency, especially when patients require hospitalization. ${ }^{2,3}$ Previous studies using protected specimen brushing have shown that bacterial infection could be the etiology in approximately $50 \%$ of acute exacerbations of COPD (AECOPD). ${ }^{4}$ However, it is sometimes difficult to distinguish colonization from infection when organisms are found in sputum cultures from COPD patients.

Recently, interest in the use of prophylactic antibiotics to prevent AECOPD has increased, ${ }^{5}$ and macrolides in this setting have the advantage of having both antibacterial and anti-inflammatory properties. Long-term macrolide therapy is routinely used in two diseases, ie, diffuse panbronchiolitis and cystic fibrosis, both of which involve chronic airway inflammation. Erythromycin is the most commonly used macrolide in diffuse panbronchiolitis, and obtains notable improvements in symptoms and survival. ${ }^{6}$ 
Studies in cystic fibrosis have mostly used azithromycin and have found improvement in lung function and a reduced exacerbation frequency. ${ }^{7,8}$ These effects are probably due to modulation of the inflammatory response and its ability to impede formation of biofilm. ${ }^{9}$ Azithromycin has also been shown to be useful in the treatment of patients with bronchiectasis and chronic bronchial infection by Pseudomonas aeruginosa..$^{10,11}$

Given the importance of inflammation ${ }^{12}$ and bacterial infection in the pathogenesis of COPD, it has been proposed that macrolides may offer unique advantages as diseasemodifying agents. Only two studies to date have analyzed the effectiveness and safety of long-term erythromycin in COPD over a 12-month period, reporting a significant reduction in moderate to severe AECOPD. ${ }^{13,14}$ It remains to be established whether the therapeutic effect of erythromycin reflects antimicrobial activity, an immunomodulatory effect, or both. Preliminary data have recently been reported for the MACRO study, a randomized controlled trial evaluating the utility of long-term azithromycin therapy to reduce AECOPD, with promising results. ${ }^{15}$ Compared with erythromycin, the prototypical 15 member-ring macrolide, azithromycin, appears to have a better safety profile in long-term use, as well as improved bacteriological activity. ${ }^{16}$

In this study, we investigated: the usefulness of long-term intermittent azithromycin therapy in reducing exacerbation frequency in severe COPD patients at a high risk of AECOPD despite conventional maximum treatment; its impact on the bacteriology of bronchial secretions, examining baseline and follow-up colonization by $P$. aeruginosa or other potentially pathogenic microorganisms; and its impact on the development of resistance to macrolides.

\section{Materials and methods Subjects}

We identified a cohort of 203 patients with severe COPD, with a postbronchodilator forced expiratory volume in one second $\left(\mathrm{FEV}_{1}\right)<50 \%$ of predicted and routinely controlled at the respiratory day care unit of the Sabadell Hospital in Barcelona, Spain, between March 2007 and August 2009. Patients with chronic bronchitis who had repeated AECOPD (at least four exacerbations in the previous year) or chronic bronchial colonization by $P$. aeruginosa treated with longterm azithromycin therapy were recruited for this study. Patients with asthma, significant bronchiectasis, malignancy, unstable heart disease, or liver disease were excluded. Ethical permission for the study was obtained from the Sabadell Hospital ethics committee.

\section{Study design}

Retrospective analysis of data for the year before initiation of long-term azithromycin therapy (for 12 months) in patients with severe COPD was undertaken in order to assess the clinical benefits of this treatment to reduce AECOPD frequency. Azithromycin (one $500 \mathrm{mg}$ tablet) was administered three times per week (Monday, Wednesday, Friday), in accordance with standard practice in patients with bronchiectasis associated with cystic fibrosis and chronic bronchial colonization by $P$. aeruginosa. ${ }^{17}$

Patients were classified into three groups according to the potentially pathogenic microorganisms isolated from their sputum samples during AECOPD in the year prior to azithromycin therapy, ie: Group 1, patients with at least two positive cultures for common potentially pathogenic microorganisms (Haemophilus influenzae, Streptococcus pneumoniae, Moraxella catarrhalis); Group 2, patients with chronic bronchial colonization by $P$. aeruginosa (at least three consecutive sputum cultures for potentially pathogenic microorganisms during a 6-month period of stability), ${ }^{18}$ and Group 3, patients whose cultures alternated between being positive for common potentially pathogenic microorganisms and $P$. aeruginosa during exacerbations but without chronic bronchial colonization by this bacteria.

We compared the following parameters overall and by group before and after the 12-month azithromycin treatment period: number of registered AECOPD; number of hospitalizations due to respiratory disease; and length of hospital stay. We also traced the evolution of positive cultures for potentially pathogenic microorganisms during AECOPD in each group and the development of resistance to azithromycin before and after long-term therapy.

All patients underwent the same scheduled clinical assessments at the respiratory day care unit ${ }^{19}$ by the same team of pulmonologists before and after starting long-term azithromycin therapy. At baseline, pulmonary function tests and use of home oxygen therapy were recorded. A highresolution computed tomography of the chest was performed in all patients in a stable state to assess and quantify possible bronchiectasis. Monitoring visits were conducted every 3 months during the 2 years of study.

Number of registered AECOPD, hospitalizations due to respiratory disease, length of hospital stays, and microbiological isolates in the previous 12 months were recorded just before the initiation of azithromycin therapy and 1 year later. A sputum sample was routinely collected during each AECOPD. All patients received regular treatment with 
long-acting beta-agonists, long-acting anticholinergics, and inhaled corticosteroids during the 2 years of study.

When used in patients with chronic $P$. aeruginosa colonization, inhaled colimycin therapy was maintained during the full length of the study and recorded. During follow-up, an electrocardiogram, liver and renal function test, and complete blood count measurements were performed before and after initiation of azithromycin treatment. The appearance of possible adverse events was assessed at each monitoring visit.

\section{Assessment of bronchiectasis}

Bronchiectasis in the high-resolution computed tomography scan was scored in each lobe by consensus, using the grading system proposed by Smith et $\mathrm{al}^{20}$ as follows: 0 if no bronchiectasis was present, 1 if $<25 \%$ of bronchi were bronchiectatic, 2 if $2 \%-49 \%$ bronchi were bronchiectatic, 3 if $50 \%-74 \%$ bronchi were bronchiectatic, and 4 if $\geq 75 \%$ of the bronchi were bronchiectatic. The lingula was graded as a separate lobe, resulting in a maximum score of 24 per patient. Patients with a score $\leq 1$ were considered normal.

\section{Definition and treatment of exacerbations}

AECOPD was defined as a sustained worsening of the patient's condition from the stable state and beyond normal day-to-day variations, of acute onset, and requiring a change in regular treatment (oral corticosteroids and/or antibiotics and/or hospital admission) in a patient with underlying COPD. $^{21}$

A chest radiography was performed for each AECOPD to exclude acute pneumonia. AECOPD were attended from Monday to Friday ( 8 am to $5 \mathrm{pm}$ ) at the respiratory day care unit, always by the same team of pulmonologists. ${ }^{19}$ Outside these hours, AECOPD were attended at the emergency department and registered by reviewing the emergency reports.

Antibiotics were prescribed during AECOPD according to Anthonisen's criteria and were adjusted by antibiogram, maintaining azithromycin at the same dose. ${ }^{22}$ Indications of hospital admission were made according to clinical practice guidelines. $^{23}$

\section{Bacteriological assessments}

Sputum samples were collected from all patients for each AECOPD and were processed locally for Gram stain and bacteriological culture. Identification and antibiogram were performed on all bacterial isolates following standardized microbiological protocols. ${ }^{24}$ Positive cultures for
P. aeruginosa during AECOPD were followed by performing additional sputum cultures at every follow-up visit to identify chronic colonization, diagnosed when three or more consecutive sputum cultures for this potentially pathogenic microorganism were found over a period of 6 months in clinically stable patients, in samples separated by at least 1 month. ${ }^{18}$

\section{Statistical analysis}

All the information was entered into a database and analyzed using SPSS version 17 (SPSS Inc, Chicago, IL). Quantitative variables are expressed as mean \pm standard deviation, and categorical variables as absolute and relative frequencies. The frequency and length of exacerbations and the microbiology of the sputum cultures before and after azithromycin treatment were compared. Statistical analysis was performed using the Student's $t$-test for paired data and Chi-square tests as required. All statistical tests were performed with a confidence level of $95 \%$.

\section{Results}

Twenty-four eligible patients with severe COPD and frequent AECOPD from the cohort of 203 patients controlled at the respiratory day care unit agreed to participate in this study. Baseline variables for the long-term azithromycin treatment group are shown in Table 1. Ten of 24 patients $(41.6 \%)$ had no detectable bronchiectasis on high-resolution

Table I Baseline variables of the first year of follow-up for the long-term azithromycin therapy group

\begin{tabular}{ll}
\hline $\begin{array}{l}\text { Baseline variables } \\
\text { (year I of follow-up) }\end{array}$ & $\begin{array}{l}\text { Mean } \\
\text { (SD) }\end{array}$ \\
\hline Age (years) & $70.9(7.4)$ \\
$\mathrm{FEV}_{\text {, }}$ L & $0.9(0.2)$ \\
$\mathrm{FEV}_{\text {, }} \%$ predicted & $32.2(9.3)$ \\
$\mathrm{FVC}(\mathrm{L})$ & $2.4(0.5)$ \\
$\mathrm{FEV} / \mathrm{FVC}$ (\%) & $39.6(9.6)$ \\
Number of AECOPD/previous year & $7.0(3.0)$ \\
Number of hospitalization/previous year & $3.3(2.0)$ \\
Days of hospital stay/previous year & $43.0(26.2)$ \\
& $\mathbf{n}(\%)$ \\
Very severe COPD (GOLD IV) & $\mathrm{II}(46)$ \\
Chronic bronchitis & $24(100)$ \\
Current smoker & $0(0)$ \\
Male & $24(100)$ \\
Chronic oxygen therapy & $5(20.8)$ \\
Pseudomonas aeruginosa colonization & $9(37.5)$ \\
\hline
\end{tabular}

Abbreviations: COPD, chronic obstructive pulmonary disease; AECOPD, acute exacerbation of chronic obstructive pulmonary disease; $\mathrm{FEV}_{1}$, forced expiratory volume in one second; FVC, forced vital capacity; GOLD, Global Initiative for Chronic Obstructive Lung Disease; SD, standard deviation. 
computed tomography. The median \pm standard deviation of total bronchiectasis score ${ }^{20}$ was $3.3 \pm 3.5$ (range 0-9).

After initiation of azithromycin, four patients did not complete the scheduled 12-month antibiotic treatment period. One was withdrawn for mild dyspepsia, one because of a diagnosis of malignancy during follow-up, and the other two discontinued the treatment prematurely as a personal decision in the absence of reported side effects. No significant adverse events were observed among the 20 patients who completed the 12-month treatment period. One patient died in hospital as a result of an AECOPD during the last month of follow-up. These 20 patients comprised the sample for the comparative study.

In the 12 months prior to the start of azithromycin, the 20 study participants had a total of 136 AECOPD, of which 72 $(52.9 \%)$ were severe and required hospitalization. Long-term azithromycin therapy achieved statistically significant reductions in AECOPD from 136 to 57 (a 58.9\% decrease) and hospitalizations from 72 to 28 (a $61.2 \%$ decrease) and a reduction of 18.7 days in yearly mean hospital stay due to respiratory disease (see Table 2).

On the basis of potentially pathogenic microorganisms isolated in sputum samples during the year prior to starting azithromycin, seven patients with common potentially pathogenic microorganisms were included in Group 1, nine patients with chronic bronchial colonization by $P$. aeruginosa in Group 2 (of whom five [55.5\%] were receiving inhaled colimycin therapy), and four patients with exacerbations due to common microorganisms and
P. aeruginosa in Group 3. Long-term azithromycin therapy reduced the number of $A E C O P D$, hospitalizations, and days of hospital stay in all groups. This reduction was particularly significant in Group 1 (common potentially pathogenic microorganisms) with a $70 \%$ reduction in AECOPD and hospitalizations, and a mean reduction of 25 days in mean hospital stay. In the group colonized by $P$. aeruginosa, a statistically significant reduction in AECOPD of $43.5 \%$ was observed, the number of hospitalizations fell by $47.1 \%$, and their hospital stays by $32.5 \%$, although these differences did not reach statistical significance. The group alternating between common potentially pathogenic microorganisms and $P$. aeruginosa during exacerbations showed improvements in all parameters studied, but no statistical comparisons were performed because of their small sample size (see Table 2 and Figure 1). AECOPD with sputum cultures isolating only common potentially pathogenic microorganisms fell from 31 of an overall $136(22.7 \%)$ to five of 57 (8.7\%) during long-term azithromycin therapy $(P<0.05)$.

Long-term azithromycin therapy rendered sputum cultures negative during AECOPD in nine of 20 patients during follow-up, including four from the common potentially pathogenic microorganism group, four from the $P$. aeruginosa colonization group (without any significant association with the presence of mucoid forms or inhaled colimycin therapy, data not shown), and one from the common potentially pathogenic $P$. aeruginosa group. Table 3 shows the microbiological evolution of patients

Table 2 Number of AECOPD, hospitalizations due to respiratory disease, and days of hospitalization with and without azithromycin, overall and according to the group of potentially pathogenic microorganisms isolated from sputum samples before initiation of azithromycin

\begin{tabular}{|c|c|c|c|c|c|c|}
\hline \multirow{2}{*}{$\begin{array}{l}\text { Variable } \\
\text { Overall } \\
(n=20)\end{array}$} & \multicolumn{2}{|c|}{$\begin{array}{l}0-12 \text { months } \\
\text { without azithromycin }\end{array}$} & \multicolumn{2}{|c|}{$\begin{array}{l}\text { I2-24 months } \\
\text { with azithromycin }\end{array}$} & \multirow[t]{2}{*}{$\%$ Red } & \multirow[t]{2}{*}{$P$} \\
\hline & $\frac{\text { withou }}{\text { Total }}$ & $\frac{\text { omycin }}{\text { Mean } \pm \text { SD }}$ & $\frac{\text { with a }}{\text { Total }}$ & $\frac{\text { Mean } \pm \text { SD }}{\text { Min }}$ & & \\
\hline Exacerbations (n) & 136 & $6.8 \pm 2.8$ & 57 & $2.8 \pm 2.5$ & 58.9 & 0.000 \\
\hline Hospitalizations (n) & 72 & $3.6 \pm 1.9$ & 28 & $1.4 \pm 1.5$ & 61.2 & 0.001 \\
\hline Hospital stay (days) & 874 & $43.7 \pm 21.4$ & 500 & $25.0 \pm 32.2$ & 42.8 & 0.013 \\
\hline \multicolumn{7}{|l|}{$\begin{array}{l}\text { Common PPM group } \\
(\mathrm{n}=7)\end{array}$} \\
\hline Exacerbations (n) & 63 & $9.0 \pm 2.3$ & 19 & $2.7 \pm 2.2$ & 70 & 0.00 \\
\hline Hospitalizations (n) & 29 & $4.1 \pm 2.6$ & 9 & $1.2 \pm 1.4$ & 70.8 & 0.04 \\
\hline Hospital stay (days) & 309 & $44.1 \pm 17.5$ & 133 & $19 \pm 25$ & 57 & 0.05 \\
\hline \multicolumn{7}{|c|}{$\begin{array}{l}\text { Pseudomonas aeruginosa group } \\
(\mathrm{n}=9)\end{array}$} \\
\hline Exacerbations (n) & 42 & $4.6 \pm 2.2$ & 24 & $2.6 \pm 2.0$ & 43.5 & 0.04 \\
\hline Hospitalizations (n) & 31 & $3.4 \pm 1.6$ & 17 & $1.8 \pm 1.7$ & 47.1 & 0.08 \\
\hline Hospital stay (days) & 454 & $50.4 \pm 23.9$ & 306 & $34.0 \pm 38.5$ & 32.5 & 0.23 \\
\hline
\end{tabular}

Abbreviations: AECOPD, acute exacerbation of chronic obstructive pulmonary disease; PPM, potentially pathogenic microorganisms; SD, standard deviation; $\%$ Red, percentage reduction. 


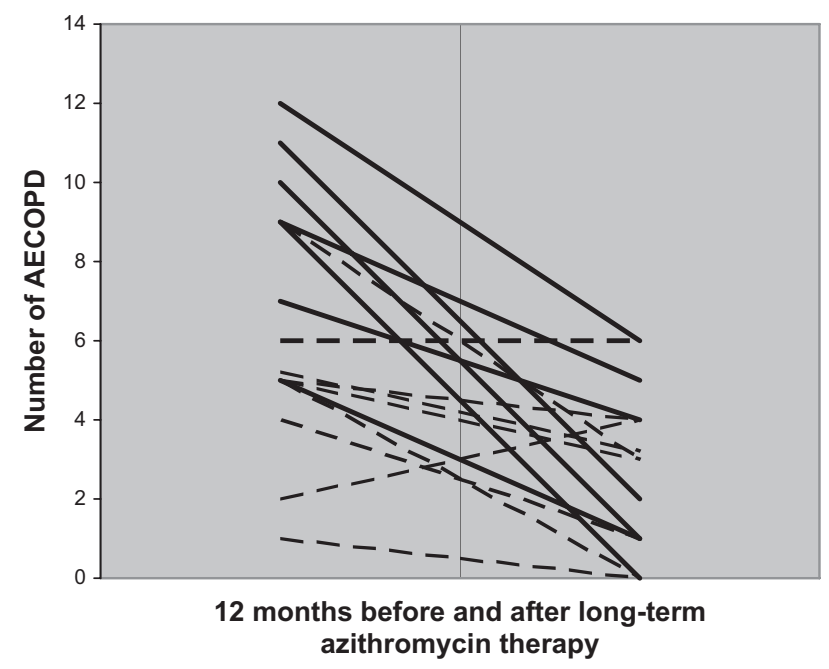

Figure I Number of AECOPD per patient before and after long-term azithromycin therapy. The continuous line represents Group I patients with potentially pathogenic microorganisms and the discontinuous line represents Group 2 patients with chronic bronchial colonization by Pseudomonas aeruginosa.

Abbreviation: AECOPD, acute exacerbation of chronic obstructive pulmonary disease.

during azithromycin treatment in relation to sputum cultures obtained during the first year.

Table 4 shows the sensitivity of common potentially pathogenic microorganisms to macrolides before and after long-term azithromycin therapy. Interestingly, all strains of H. influenzae isolated were resistant to erythromycin and $30 \%$ to clarithromycin, while none were resistant to azithromycin prior to treatment. The macrolide-resistant $S$. pneumoniae strains were also resistant to clindamycin, representing a highly resistant phenotype.

On assessment of the bactericidal effect of long-term azithromycin therapy on common potentially pathogenic microorganisms and the development of bacterial resistance, we found no isolates of $M$. catarrhalis with azithromycin resistance during follow-up, a single isolate of $H$. influenzae with resistance to the antibiotic, and four isolates of $S$. pneumoniae, all resistant to azithromycin. The prevalence of resistance did not show statistically significant differences from the figures found in the first year of the study.

\section{Discussion}

This is the first study to examine long-term intermittent azithromycin therapy over a 12-month period in patients with severe COPD and repeated AECOPD, or chronically colonized by $P$. aeruginosa, assessing its usefulness in reducing exacerbation frequency and its bactericidal effect, and comparing the results of sputum cultures at exacerbation with the prevalence of bacteria-related exacerbation before the treatment. Our results show that long-term intermittent dosing of azithromycin as addon therapy to conventional maximum triple therapy (longacting anticholinergics, long-term beta-agonists, and inhaled corticosteroids) reduces the number of AECOPD and hospitalizations by more than a half, and also the days of hospital stay. The treatment was well tolerated over the 12-month period, and was stopped by only one patient due to dyspepsia.

Few studies focusing on the prophylactic use of antibiotics in COPD to prevent AECOPD have been published thus far, most of them examining long-term use of fluoroquinolones or macrolides. Sethi et al demonstrated that a 12-month regime with pulsed moxifloxacin $400 \mathrm{mg}$ once a day for 5 days every 8 weeks achieved a $25 \%$ reduction in AECOPD, and a larger reduction (45\%) in patients who reported mucopurulent sputum at baseline. The authors concluded that pulsed moxifloxacin might be indicated in COPD patients with a high frequency of AECOPD, but excluded patients colonized by $P$. aeruginosa, one of the specific subgroups discussed in our study, because of the risk of development of resistant strains.$^{25}$ Long-term macrolide therapy in COPD was also advocated in two published studies which analyzed erythromycin over a period of 12 months, with a significant reduction in the number and severity of AECOPD. ${ }^{13,14}$ In the first, Suzuki et al reported the results of a prospective randomized trial of erythromycin therapy $200-400 \mathrm{mg}$ /day versus nonactive treatment for 12 months in 109 COPD patients

Table 3 Microbiological evolution during long-term azithromycin therapy according to baseline groups and sputum culture isolates during AECOPD

\begin{tabular}{|c|c|c|c|c|c|}
\hline \multirow{2}{*}{$\begin{array}{l}\text { Year I } \\
\text { no azithromycin }\end{array}$} & \multirow[t]{2}{*}{$\mathbf{n}$} & \multicolumn{4}{|c|}{ Year 2 azithromycin therapy } \\
\hline & & No AECOPD & $\begin{array}{l}\text { AECOPD with } \\
\text { negative cultures }\end{array}$ & $\begin{array}{l}\geq I \text { AECOPD for } \\
\text { common PPMs }\end{array}$ & $\begin{array}{l}\geq I \text { AECOPD } \\
\text { for P. aeruginosa }\end{array}$ \\
\hline Common PPM & 7 & 1 & 3 & 2 & 1 \\
\hline P. aeruginosa colonization & 9 & 2 & 2 & 0 & 5 \\
\hline Alternating PPM & 4 & 1 & 0 & 0 & 3 \\
\hline Total (patients) & 20 & 4 & 5 & 2 & 9 \\
\hline
\end{tabular}

Abbreviations: AECOPD, acute exacerbation of chronic obstructive pulmonary disease; PPM, potentially pathogenic microorganisms; P. aeruginosa, Pseudomonas aeruginosa. 
Table 4 Common microorganisms isolated in sputum culture during exacerbations before and after starting azithromycin and its antibiogram to macrolides

\begin{tabular}{|c|c|c|c|c|c|c|c|c|c|c|}
\hline \multirow{2}{*}{$\begin{array}{l}\text { Year I } \\
\text { no AZT }\end{array}$} & \multirow[t]{2}{*}{ Isolates pre-AZT } & \multicolumn{3}{|c|}{ Erythromycin } & \multicolumn{3}{|c|}{ Clarithromycin } & \multicolumn{3}{|c|}{ Azithromycin } \\
\hline & & Sen & Int & Res & Sen & Int & Res & Sen & Int & Res \\
\hline H. influenzae & $10(31.3 \%)$ & 0 & 0 & 10 & 7 & 2 & I & 10 & 0 & 0 \\
\hline S. pneumoniae & $6(18.8 \%)$ & 3 & 0 & 3 & 3 & 0 & 3 & 3 & 0 & 3 \\
\hline M. catarrhalis & I5 (50\%) & 14 & I & I & 15 & 0 & 1 & 15 & 0 & 0 \\
\hline Year 2 & Isolates post AZT & & & & & & & & & \\
\hline \multicolumn{11}{|l|}{ AZT therapy } \\
\hline H. influenzae & I (20\%) & 0 & 0 & I & 0 & 0 & 1 & 0 & 0 & I \\
\hline S. pneumoniae & $4(80 \%)$ & 0 & 0 & 4 & 0 & 0 & 4 & 0 & 0 & 4 \\
\hline M. catarrhalis & 0 & 0 & 0 & 0 & 0 & 0 & 0 & 0 & 0 & 0 \\
\hline
\end{tabular}

Abbreviations: AZT, azithromycin; H. influenzae, Haemophilus influenzae; S. pneumoniae, Streptococcus pneumoniae; M. catarrhalis, Moraxella catarrhalis; SEN, sensitive; INT, intermediate; RES, resistant.

(average $\mathrm{FEV}_{1} 1.4 \mathrm{~L}$ ). ${ }^{13}$ They found a statistically significant increase in the relative risk of AECOPD in the control group versus the erythromycin group of 4.71 (95\% CI, 1.53-14.5; $P=0.007)$ and more severe AECOPD in the control group than in the erythromycin group $(P=0.0007)$. In the second, a single-center, randomized, controlled trial, Seemungal et al administered erythromycin $250 \mathrm{mg}$ twice daily or placebo for 1 year to 109 patients with moderate to severe COPD (mean FEV $150 \%$ of predicted). ${ }^{14}$ Erythromycin reduced AECOPD in relative terms by $35 \%$ and increased the median time to first exacerbation from 89 to 271 days, both differences being statistically significant.

Preliminary data from the prospective, placebo-controlled MACRO study in 1142 COPD patients randomized to receive azithromycin $250 \mathrm{mg}$ or placebo daily for 1 year have been promising. ${ }^{15}$ Inclusion criteria were use of supplemental oxygen or treatment with systemic steroids for an AECOPD within the previous year. The frequency of AECOPD for those receiving azithromycin was lower at 1.4 versus 1.8 patients/year $(P=0.004)$.

The microbiology data collected from the MACRO study have not yet been formally published.

Neither study assessed the impact of treatment in patients colonized by $P$. aeruginosa. Seemungal et al included patients with moderate to severe COPD in their series, of whom only $35 \%$ had three or more AECOPD during the year prior to inclusion. In the MACRO study, the frequency of AECOPD was also low. Our study enrolled an homogeneous population sample of severe or very severe COPD with very frequent AECOPD and patients with chronic colonization by $P$. aeruginosa

Azithromycin offers clinical advantages over erythromycin. Its metabolism does not interfere with the metabolic pathway of cytochrome P450, thus avoiding possible metabolic interference with other drugs often used in
COPD which share the same pathway, such as steroids and theophylline. It has better gastrointestinal tolerance and less hepatotoxicity, and because it is not associated with long QT syndrome, is better tolerated and has a better safety profile in long-term use. ${ }^{26}$ Even though macrolides provide adequate coverage for the most frequent potentially pathogenic microorganisms identified in AECOPD, their activity against $H$. influenzae differs. Azithromycin, the prototypical 15-member ring macrolide, has greater bacteriological and clinical activity than 14-membered ring macrolides, such as erythromycin and clarithromycin. ${ }^{27}$ Interestingly, in our study, all strains of $H$. influenzae isolated prior to the initiation of long-term azithromycin were resistant to erythromycin and $30 \%$ were resistant to clarithromycin, while none was resistant to azithromycin.

The subanalysis shows that Group 1, despite being the one with the highest number of AECOPD and hospitalizations prior to azithromycin therapy, demonstrated the greatest improvement with long-term azithromycin treatment, with highly significant reductions of around $70 \%$ in AECOPD and hospitalizations. The mechanism of improvement may be related to the antibacterial activity of azithromycin, particularly with regard to $H$. influenzae and $M$. catarrhalis. AECOPD with isolates positive for these potentially pathogenic microorganisms fell from 25 pre-azithromycin to just one (for $H$. influenzae) during long-term azithromycin therapy. However, we did not observe this level of bacterial eradication for S. pneumoniae, which has a higher prevalence of resistance to azithromycin, independently of the use of this antibiotic in the study.

We cannot exclude the possibility that the improvement observed may be due in part to the anti-inflammatory and immunomodulatory properties of azithromycin. ${ }^{9}$ Azithromycin may decrease sputum volume and its viscoelas- 
ticity, and increase mucociliary transport. ${ }^{28}$ Azithromycin also accumulates in neutrophils, interfering with chemotaxis to the inflammatory focus and promoting neutrophil apoptosis and clearance by macrophages. ${ }^{29}$ In their trial with long-term erythromycin, Seemungal et al analyzed inflammatory mediators in sputum (interleukin-6, interleukin-8, myeloperoxidase) and plasma (interleukin-6, interleukin-8, C-reactive protein) as secondary outcomes, but found no statistically significant treatment-related differences. ${ }^{14}$ The lack of effect of erythromycin on inflammatory markers suggests that the antimicrobial effects of macrolides in the treatment of COPD patients may be more important.

Patients with advanced COPD, especially those with repeated courses of antibiotic therapy or oral corticosteroids and requiring hospital admissions, have an increased risk of exacerbations caused by $P$. aeruginosa. ${ }^{30,31}$ In these patients, antibiotic treatment decisions should consider both the severity of AECOPD and the risk for isolation of P. aeruginosa. ${ }^{23}$

The fact that $45 \%$ of our patients had chronic bronchial colonization by P. aeruginosa (Group 2) reflects the severity of their COPD. In these patients, long-term azithromycin therapy also improved all the outcomes analyzed, achieving a statistically significant reduction of $43 \%$ in AECOPD. Hospitalizations and days of hospital stay also fell to $47 \%$ and $32 \%$, respectively, in these patients, although these differences did not reach statistical significance.

Macrolides, especially azithromycin, are useful in the treatment of chronic bronchial colonization by $P$. aeruginosa in patients with bronchiectasis, mainly in cases associated with cystic fibrosis. ${ }^{7,32}$ Azithromycin interferes with production of virulence factors by $P$. aeruginosa, reduces biofilm formation by inhibiting alginate production, and decreases bacterial adherence. ${ }^{33}$ For these reasons, we think that longterm azithromycin is especially indicated in these patients. However, the reductions in hospitalizations and days of mean hospital stay are less evident, possibly because they often require prolonged hospitalizations for parenteral antibiotic treatment, because $P$. aeruginosa is often resistant to oral antibiotics in this clinical situation.

Potential limitations of our study are the small number of patients included and the absence of a control group. However, our findings are very promising, especially for selected patients with severe COPD at high risk of exacerbations despite conventional maximum treatment. This study may help in the design of future, randomized, controlled trials including more patients and assessing the impact of treatment in patients with different degrees of severity, focusing on aspects such as efficacy, safety, development of microbiological resistance, or economic burden.

In conclusion, we have shown that long-term intermittent azithromycin therapy, administered three times a week at a dosage of $500 \mathrm{mg}$, is well tolerated and associated with significant reductions in AECOPD, number of hospitalizations, and days of hospital stay in patients with severe COPD and repeated AECOPD. This improvement is especially significant in patients with AECOPD associated with common potentially pathogenic microorganisms, possibly due to the antibacterial activity of the drug, and in patients with chronic bronchial colonization by $P$. aeruginosa due to its ability to prevent AECOPD for common potentially pathogenic microorganisms also in these patients. These results suggest that long-term intermittent azithromycin therapy may be useful in the treatment of patients with severe COPD and frequent exacerbations.

\section{Disclosure}

The authors report no conflicts of interest in this work. Funded in part by Fondo de Investigaciones Sanitarias and Ciber de Enfermedades Respiratorias - CibeRes.

\section{References}

1. Hurst JR, Vestbo J, Anzueto A, et al. Evaluation of COPD Longitudinally to Identify Predictive Surrogate Endpoints (ECLIPSE) Investigators. Susceptibility to exacerbation in chronic obstructive pulmonary disease. N Engl J Med. 2010;363:1128-1138.

2. Soler-Cataluna JJ, Martínez MA, Román P, Salcedo E, Navarro M, Ochando R. Severe acute exacerbations and mortality in patients with chronic obstructive pulmonary disease. Thorax. 2005;60:925-931.

3. Moreno A, Belmonte Y, Gallego M, Pomares X, Real J. [Causes of death and risk factors for mortality in patients with severe chronic obstructive pulmonary disease.] Arch Bronconeumol. 2009;45:181-186. [Spanish.]

4. Monsó E, Ruiz J, Rosell A, et al. Bacterial infection in chronic obstructive pulmonary disease. A study of stable and exacerbated outpatients using the protected specimen brush. Am J Respir Crit Care Med. 1995; 152:1316-1320.

5. Kunisaki KM, Niewoehner DE. Antibiotic prophylaxis for chronic obstructive pulmonary disease: Resurrecting an old idea. Am J Respir Crit Care Med. 2008;178:1098-1099.

6. Kudoh S, Azuma A, Yamamoto M, Izumi T, Ando M. Improvement of survival in patients with diffuse panbronchiolitis treated with low-dose erythromycin. Am J Respir Crit Care Med. 1998;157:1829-1832.

7. Southern KW, Barker PM, Solis A. Macrolide antibiotics for cystic fibrosis. Cochrane Database Syst Rev. 2004:CD002203.

8. Clement A, Tamelet A, Leroux E, Ravilly S, Fouroux B, Jais JP. Longterm effects of azithromycin in patients with cystic fibrosis: A double blind, placebo controlled trial. Thorax. 2006;61:895-902.

9. Shinkai M, Henke MO, Rubin BK. Macrolide antibiotics as immunomodulatory medications: Proposed mechanisms of action. Pharmacol Ther. 2008;117:393-405.

10. Wolter J, Seeney S, Bell S, Bowler S, Masel P, McCormack J. Effect of long term treatment with azithromycin on disease parameters in cystic fibrosis: A randomised trial. Thorax. 2002;57:212-216.

11. Saiman L, Marshall BC, Mayer-Hamblett N, et al; Macrolide Study Group. Azithromycin in patients with cystic fibrosis chronically infected with Pseudomonas aeruginosa: A randomized controlled trial. JAMA. 2003;290:1749-1756. 
12. Gan WQ, Man SF, Senthilselvan A, Sin DD. Association between chronic obstructive pulmonary disease and systemic inflammation: A systematic review and meta-analysis. Thorax. 2004;59:574-580.

13. Suzuki T, Yanai M, Yamaya M, et al. Erythromycin and common cold in COPD. Chest. 2001;120:730-733.

14. Seemungal TA, Wilkinson TM, Hurst JR, Perera WR, Sapsford RJ, Wedzicha JA. Long-term erythromycin therapy is associated with decreased chronic obstructive pulmonary disease exacerbations. Am J Respir Crit Care Med. 2008;178:1139-1147.

15. Albert RK, Bailey WC, Casaburi R, et al. Chronic azithromycin decreases the frequency of chronic obstructive pulmonary disease exacerbations. Abstract A6416 presented at the American Thoracic Society Congress, May 13-18, 2011, Denver, CO.

16. Cobos-Trigueros N, Ateka O, Pitart C, Vila J. [Macrolides and ketolides.] Enferm Infecc Microbiol Clin. 2009;27:412-418. [Spanish.]

17. Flume PA, O’Sullivan BP, Robinson KA, et al. Cystic Fibrosis Foundation. Pulmonary Therapies Committee Cystic fibrosis pulmonary guidelines: Chronic medications for maintenance of lung health. Am J Respir Crit Care Med. 2007;176:957-969.

18. Vendrell M, de Gracia J, Olveira C, et al. [Diagnosis and treatment of bronchiectasis. Spanish Society of Pneumology and Thoracic Surgery.] Arch Bronconeumol. 2008;44:629-640. [Spanish.]

19. Pomares X, Monton C. Respiratory day hospital: What have we learned? Med Clin (Barc). 2011;136:454-455.

20. Smith IE, Jurriaans E, Diederich S, Ali N, Shneerson J, Flower CDR. Chronic sputum production: Correlation between clinical features and findings on high resolution computed tomographic scanning of the chest. Thorax. 1996;51:914-918.

21. Rodriguez-Roisin R. Toward a consensus definition for COPD exacerbations. Chest. 2000;117:398-401.

22. Anthonisen NR, Manfreda J, Warren CPW, Hershfield ES, Harding GKM, Nelson NA. Antibiotic therapy in exacerbations of chronic obstructive pulmonary disease. Ann Intern Med. 1987;106:196-204.

23. Pauwels RA, Buist AS, Calverley PM, Jenkins CR, Hurd SS. Global strategy for the diagnosis, management, and prevention of chronic obstructive pulmonary disease. NHLBI/WHO Global Initiative for Chronic Obstructive Lung Disease (GOLD) Workshop summary. Am J Respir Crit Care Med. 2001;163:1256-1276.
24. Clinical and Laboratory Standards Institute. Performance Standards for Antimicrobial Susceptibility Testing; Twentieth Informational Supplement. Wayne, PA: Clinical and Laboratory Standards Institute; 2010.

25. Sethi S, Jones PW, Theron MS, et al; PULSE Study Group. Pulsed moxifloxacin for the prevention of exacerbations of chronic obstructive pulmonary disease: A randomized controlled trial. Respir Res. 2010; $11: 10$.

26. Huang BH, Wu CH, Hsia CP, Yin Chen C. Azithromycin-induced torsade de pointes. Pacing Clin Electrophysiol. 2007;30:1579-1582.

27. Martinez FJ, Curtis JL, Albert R. Role of macrolide therapy in chronic obstructive pulmonary disease. Int J Chron Obstruct Pulmon Dis. 2008;3: 331-350.

28. Tamaoki J, Takeyama K, Tagaya E, Konno K. Effect of clarithromycin on sputum production and its rheological properties in chronic respiratory tract infections. Antimicrob Agents Chemother. 1995;39: 1688-1690.

29. Parnham MJ, Culić O, Eraković V, et al. Modulation of neutrophil and inflammation markers in chronic obstructive pulmonary disease by shortterm azithromycin treatment. Eur J Pharmacol. 2005;517:132-143.

30. Garcia-Vidal C, Almagro P, Romaní V, et al. Pseudomonas aeruginosa in patients hospitalised for COPD exacerbation: A prospective study. Eur Respir J. 2009;34:1072-1078.

31. Miravitlles M, Espinosa C, Fernández-Laso E, Martos JA, Maldonado JA, Gallego M. Relationship between bacterial flora in sputum and functional impairment in patients with acute exacerbations of COPD. Study Group of Bacterial Infection in COPD. Chest. 1999; 116:40-46.

32. Southern KW, Barker PM. Azithromycin for cystic fibrosis. Eur Respir J. 2004;24:834-838.

33. Tateda K, Ishii Y, Kimura S, Horikawa M, Miyairi S, Yamaguchi K. Suppression of Pseudomonas aeruginosa quorum-sensing systems by macrolides: A promising strategy or an oriental mystery? J Infect Chemother. 2007;13:357-367.
International Journal of COPD

\section{Publish your work in this journal}

The International Journal of COPD is an international, peer-reviewed journal of therapeutics and pharmacology focusing on concise rapid reporting of clinical studies and reviews in COPD. Special focus is given to the pathophysiological processes underlying the disease, intervention programs, patient focused education, and self management protocols.

\section{Dovepress}

This journal is indexed on PubMed Central, MedLine and CAS. The manuscript management system is completely online and includes a very quick and fair peer-review system, which is all easy to use. Visit http://www.dovepress.com/testimonials.php to read real quotes from published authors. 